\title{
Motion Retargetting and Transition in Different Articulated Figures
}

\author{
Ming-Kai Hsieh* \\ Bing-Yu Chen ${ }^{\dagger}$ \\ National Taiwan University \\ *lionkid@cmlab.csie.ntu.edu.tw †robin@ntu.edu.tw
}

Ming Ouhyoung ${ }^{\ddagger}$

${ }^{\ddagger}$ ming@csie.ntu.edu.tw

\begin{abstract}
In this paper, we present an interactive system to transfer and concatenate motions in different articulated figures, such as human and dog. This system first constructs a union skeleton that contains both bone structures with bone correspondence manually assigned by users, then aligns the initial poses of these two skeletons. With the initial poses aligned, motion data can be transferred between them. In addition, by blending similar poses of different skeletons, we can produce a seamless transition sequence which can be used to drive a meta-mesh and generate an animated morphing result.
\end{abstract}

\section{Introduction}

The process of animation creation is notorious for its timeconsuming and tedious manual tweaking, and the results are often not good enough. Thus, how to reuse an existing motion (keyframe animation or motion capture data) is an important topic. It is a useful technique for user to produce a base motion from another motion, then add some characteristics by keyframing. Recently, with the advance and prevalence of motion capture techniques, more and more high quality motion data can be obtained from the world wide web or commercial companies. There are three main techniques: first, motion retargetting, which allows user to adapt a motion to a different character; second, motion synthesis, which combines two or more motions together to produce a new motion; third, motion transition, which generates a smooth transition between two motions that concatenates them seamlessly. But reusing these motion data on another character is not a trivial task, especially when two characters are different.

If we want a human character to imitate the walking motion of a dog, or a flying motion of a bird, the problems we will encounter are: they may have different number of bones, initial poses, or topologies. Same problems (except for the topologies problem) can occur even if we are dealing with same type of characters. Different motion capture companies may define different human skeletons; different artists tend to define different animal skeletons according to their experiences. So if we can transfer motions between two different characters, we can save the animation production time. Besides, transition between two motions of different skeletons will produce an animated morphing result, such as a walking human morph to a running dog. This technique may be useful in game or movie industries.

\subsection{Overview}

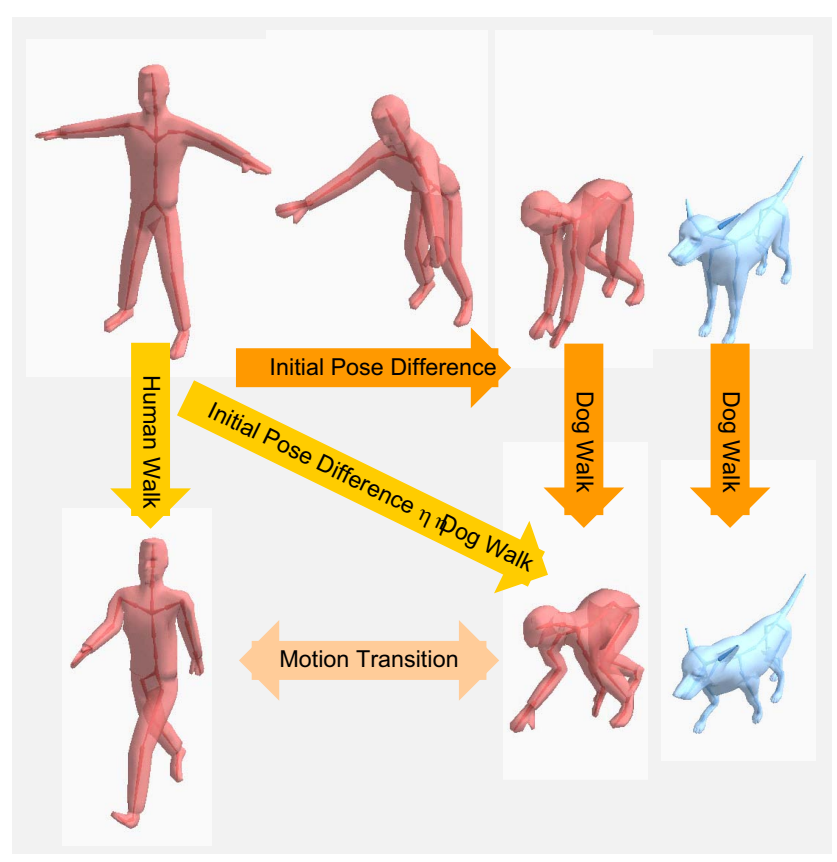

Figure 1: An overview of our system. The source skeleton is a dog, and the target skeleton is a man.

In order to accomplish motion retargetting and transition in different skeletons, we have to define the correspondence of the source and target skeletons. Then after aligning their initial poses, we can retarget the motion data between them. Moreover, motion transition can be done by constructing a meta-skeleton which contains both skeleton structures, then blending similar frames and bone lengths of these two motions. Figure 1 is an overview of our system. In this fig- 


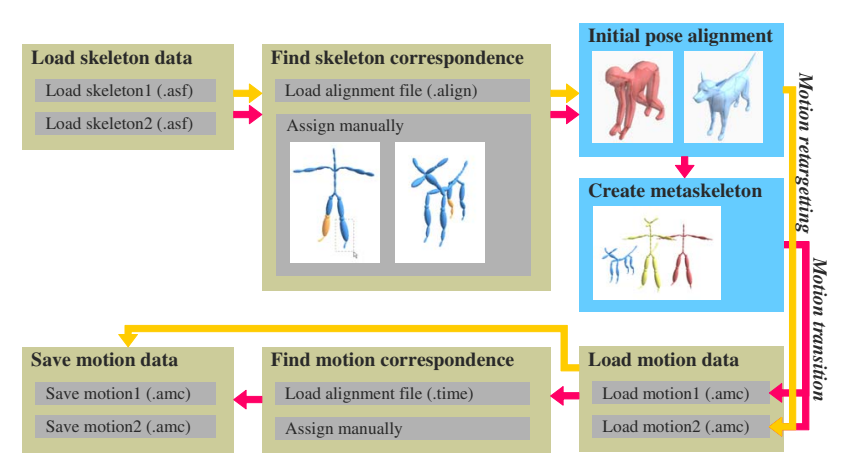

Figure 2: System workflow.

ure, the source skeleton is a dog, and the target skeleton is a man. We can retarget the dog's walking motion to the man by adding their initial pose difference and dog's walking motion on the man's character. Then we can concatenate the man's and the dog's walking motions by their meta-skeleton. Figure 2 shows the system workflow. The remainder of this paper are organized as follows: Section 2 reviews some previous motion retargetting and transition techniques. Sections 3 and 4 focus on the main algorithm. Section 5 shows several animation snapshots produced by our system. In Section 6, we conclude this paper and list some future work.

\section{Related Work}

Gleicher [5] exploited spacetime constraints to take the spatial and temporal constraints of the original motion into account. He formulates motion retargetting as a big constraints optimization problem, and tried to reduce the computational costs. In his paper, he also discussed the issues of transferring motion between different characters. Using the same spacetime constraints technique, they can retarget a human walking motion to a rigid can. Popovic̀ and Witkin [10] also use spacetime constraints scheme to solve motion transformation problem with taking dynamics into account. Their method can also map motion between different kinematic structures. The main idea of their method is to manually simplify the original skeleton to a reduced skeleton, and fit the original motion data to it. The computational cost of spacetime constraints editing or transformation can be reduced by using this simplified model. After editing, they fit the result motion back to the original skeleton. Lee and Shin [9] develop an interactive system to adapt existing human character motions to have desired features by fitting a set of constraints induced by both characters and environments. They fuse a hierarchical curve fitting technique with their own inverse kinematics solver to accomplish the tasks. In contrast to [5], who solve motion retargetting problem in a large non-linear optimization problem, they decouple this problem into several manageable subproblems that can be efficiently solved.

The goal of motion transition is to seamlessly concatenate two motions. Most previous techniques are focusing on transiting motions of the same skeleton. First, the similar frames of these two motions are found by some distance metrics. These frames are regarded as good transition points. Then the neighbors of corresponding frames are blended according to some ease-in ease-out blend weights to produce a smooth result.

Bruderlin and Williams [4] use dynamic programming and timewarping methods to produce transitions. They treat motions as time-varying continuous functions and apply signal processing techniques for motion blending and transition. After finding the similar frames, displacement mapping is used to combine two motion without losing their continuous characteristics. Several work like $[12,6,8]$ also use displacement mapping techniques to blend motions. Unuma et al. [11] use fourier function approximation to represent a rotation angle of a bone in a period of time. The transition of two motions of the same character can be produced by interpolating the fourier parameters in frequency domain and then transforming back to spatial domain. Ashraf and Wong [1] propose a semi-automatic labeling technique to find out the correspondence of two motions by analyzing the end-effector acceleration zero-crossings of user-specified joints. Their motion-state based transition can handle more categories of motions than other signal based techniques, because the latter methods often blend the whole body movement, unlike their decoupled interpolation approach, which consider upper and lower halves apart. Later, they add inverse kinematics constraints to their system for correcting foot sliding problems on the fly $[2,3]$.

\section{Motion Retargetting}

A skeleton $S$ is defined as a collection of several bones $b_{i} \in B$ and a motion clip $M_{S}(t)$ is recorded by the state of each bone (included its translation and rotation angels) in time $t$, relative to its initial pose. Thus, to retarget motions between different skeletons $S$ and $S^{\prime}$ (Figure 3), a straightforward idea is to align their initial pose first, then transfer the motion data from one to the other. First, we need a function to describe the corresponding bones in $S$ and $S^{\prime}$. Since the initial pose of two skeletons may be different, we have to align these initial pose in the beginning.

\subsection{Skeleton Correspondence}

In our system, users can assign three types of correspondence manually through our interface: (1) one-to-one correspondence (occurred in most cases), (2) many-to-one correspondence (e.g. we may map two legs of a human to the 


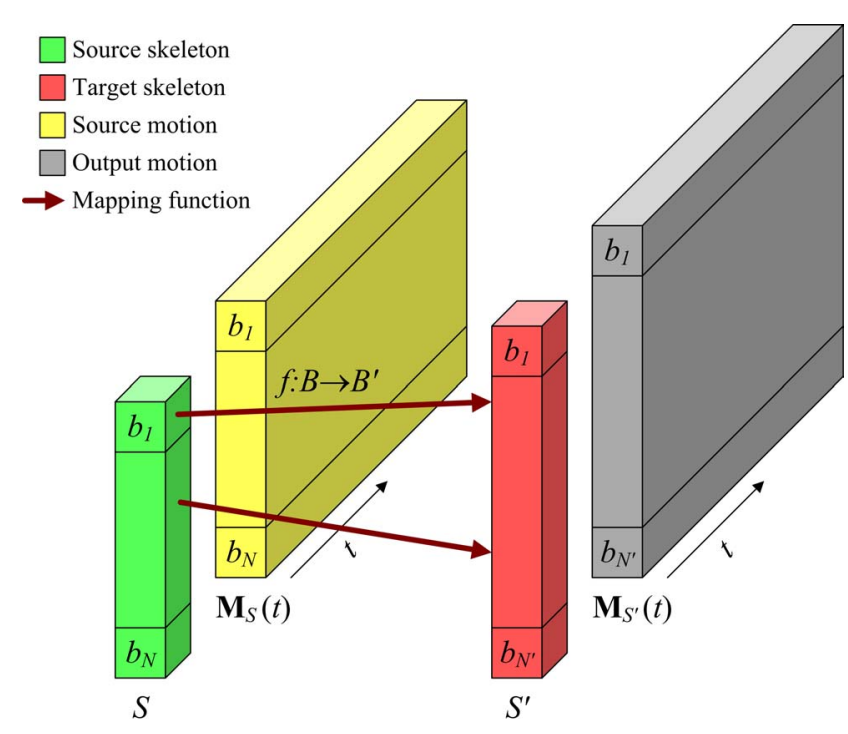

Figure 3: An illustration of motion retargetting.

tail fin of a fish), and (3) no correspondence (e.g. we cannot map a tail of a dog to any bone of a human). Figure 4 shows an example of skeleton correspondence of a dog and a human. Because we are dealing with different skeletons, fully automatic skeleton matching techniques which consider the bone direction, topologies or dominance (the number of successors) cannot work in the most cases. In addition, we want to provide the flexibility for artists to retarget motions in their own will, so user intervention is still needed. Other semi-automatic matching techniques like [13] can be added in this system.

\subsection{Initial Pose Alignment}

To align the initial pose of two skeletons, we recursively count the rotation angle between two corresponding bones from root to leaf and directly apply the results to the target skeleton before computing the next angle difference. This method can only guarantee the bone directions are aligned, we do not consider bone twist effect, so unexpected results can occur, such as the orientation of the human's hand shown in Figure 5. That is because that there is no finger map to dog's limbs. In our current implementation, the user can adjust the bone twist through our interface.

\subsection{Motion Data Transfer}

With the initial poses of the two skeletons are aligned, we can transfer motion data between each other, but the local frames of corresponding bones may be different (Figure 5). In this case, we can transform the source rotation angles from local to global coordinate, then transform back to the target's local coordinate.

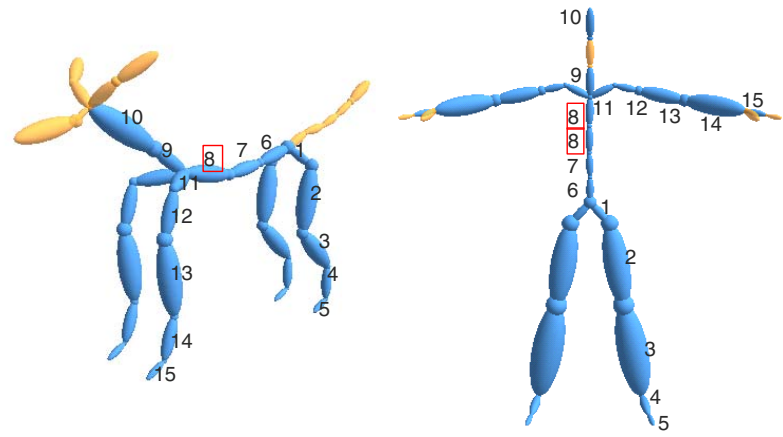

Figure 4: Skeleton correspondence of a dog and a human. Corresponding bones are represented by the same number. Numbers enclosed with red squares represents many-to-one correspondence. Orange bones denote bones that have no correspondence.

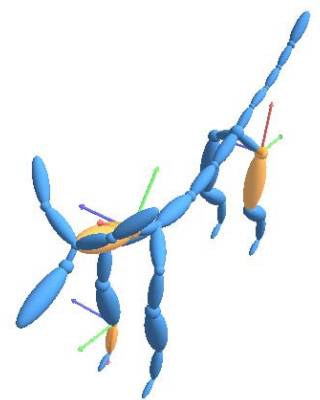

(a) A skeleton of dog.

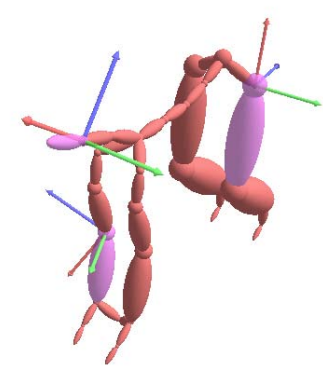

(b) A skeleton of human with initial pose aligned with a dog.
Figure 5: Local frames of the corresponding bones may not be the same even the initial poses are aligned. The highlighted bones denote the corresponding bones, and the $\mathrm{x}, \mathrm{y}$, $\mathrm{z}$ axes are colored by red, green, blue, respectively.

\section{Motion Transition}

Our system provides the ability to transfer the motions in different skeletons by the bone correspondences. We also want to know how to transit the motions between different skeletons. For example, morph a running human to a flying bird. However, if two mesh models are parameterized with their initial pose aligned, we can morph the meta-mesh between these two models while it is animated by a metaskeleton that contains both skeletons. In this paper, we only discuss skeleton morphing. The main idea of motion transition in different skeletons is as follows: (1) first we construct a meta-skeleton that contains both skeletons by bone correspondence information; (2) then we retarget the source motion to target with the techniques described in Section 3; 
(3) finally with the correspondence of these two motions are found, we can use motion blending to produce a skeleton morphing result.

\subsection{Meta-Skeleton Construction}

After the bone correspondence of the input skeletons are found, we can construct a meta-skeleton (union skeleton) that contains both information of input skeletons. Without meta-skeleton, we cannot generate a consistent transition result in different skeletons, because there may be several bones that have no correspondence. Figure 6 shows a metaskeleton construct by a dog and a human. Sometimes the bone correspondence may produce ambiguity in the metaskeleton, we have to prevent users from assigning ambiguous bone correspondence in the bone corresponding interface.

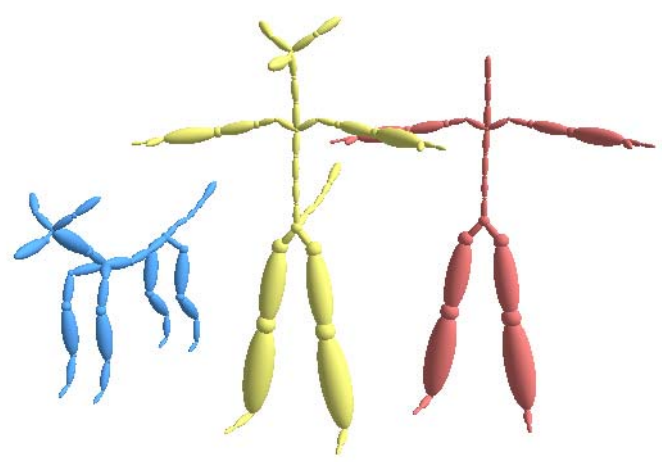

Figure 6: A meta-skeleton (the yellow one) constructed by a dog and a human.

\subsection{Motion Correspondence}

In order to get a better transition result, we need to find the correspondence between two motions. Blending frames with similar poses can produce a smoother transition. However, since we are dealing with two different motions in different skeletons, even if they do the same type of motion (such as walking), their postures will remain different. In this case, signal based or joint based motion correspondence approaches may fail. Signal based techniques [4] are often short of establishing meaningful correlation between motions that have many DOFs (degree of freedoms), since they only consider one DOF at a time. Joint position based approaches [7] take the whole body motion into account, but lack of the abilities to find correspondence between different skeletons or dissimilar motions. In current imple- mentation, we provide an interface for users to assign the corresponding frames manually.

\section{Results}

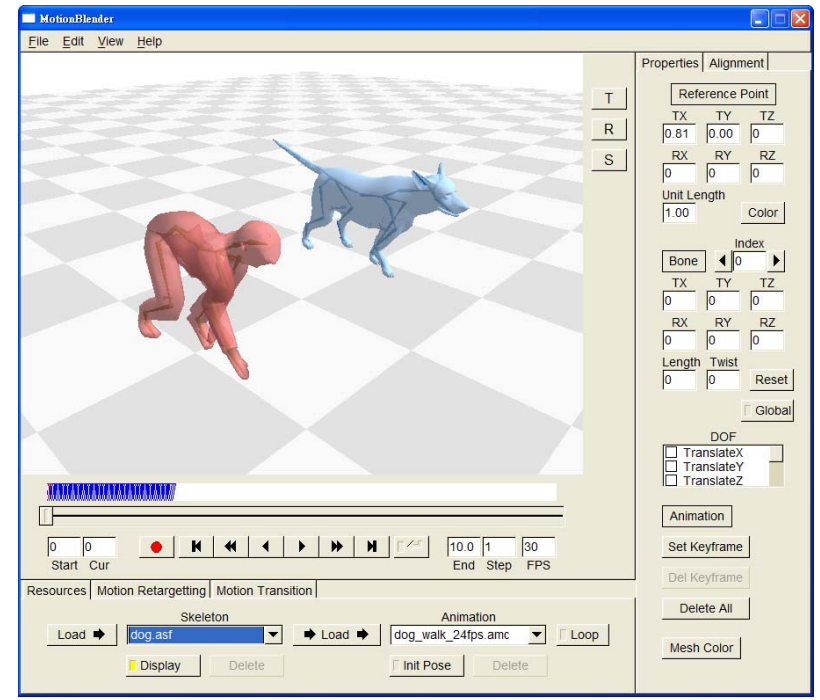

Figure 7: The interface of our system.

Figure 7 shows the interface of our system, which is developed with FLTK ${ }^{1}$. The results of motion retargetting and transition are shown in Figures 8 and 9, respectively.

\section{Conclusions}

In this paper, we presented a system that can perform motion retargetting and transition with different skeletons. After setting the skeleton correspondence by users, the initial poses are automatically aligned, then the motion data are transferred directly. In addition, if both motions of two input skeletons are provided, we can blend corresponding frames and bone length to produce a skeleton morphing result. However, there are still lots of work to do, including:

- The joint constraints of the retargetted skeleton must be taken into consideration to produce a plausible retargetting result.

- Spacetime constraints and inverse kinematics should be considered to correct the path of the motion and other external constraints (constraints can be manually set by users).

- Collision detection should also be taken into account to prevent unnatural intersection of the body and limbs.

${ }^{1}$ http://www.fltk.org/ 


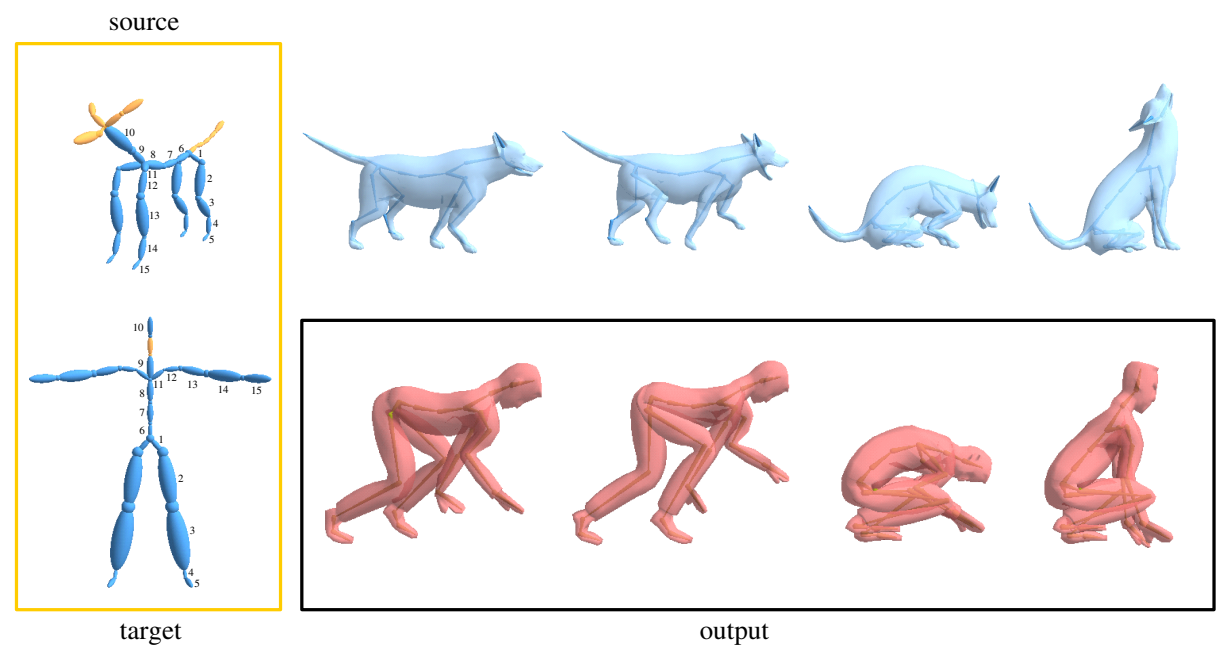

(a) Retarget a dog's motion to a man.

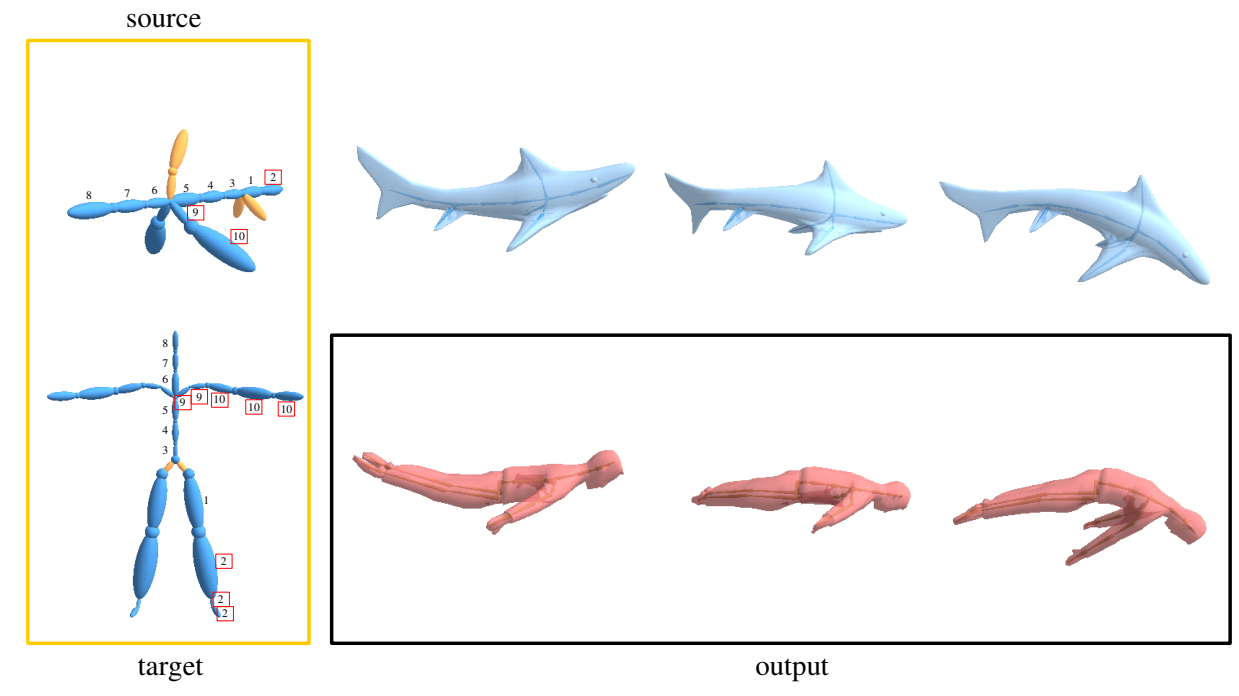

(b) Retarget a shark's motion to a man.

Figure 8: The motion retargetting results of our system.

\section{Acknowledgments}

This work was partially supported by the National Science Council of Taiwan under the contract numbers: 93-2213-E002-084, and 94-2213-E-002-097.

\section{References}

[1] Golam Ashraf and Kok Cheong Wong. Generating consistent motion transition via decoupled framespace interpolation. Computer Graphics Forum (Eurographics 2000 Conference Proceedings), 19(3):447-456, 2000.
[2] Golam Ashraf and Kok Cheong Wong. Constrained framespace interpolation. In Proceedings of Computer Animation 2001, pages 61-72, 2001.

[3] Golam Ashraf and Kok Cheong Wong. Semantic representation and correspondence for state-based motion transition. IEEE Transactions on Visualization and Computer Graphics, 9(4):481-499, 2003.

[4] Armin Bruderlin and Lance Williams. Motion signal processing. In ACM SIGGRAPH 1995 Conference Proceedings, pages 97-104, 1995.

[5] Michael Gleicher. Retargetting motion to new characters. In ACM SIGGRAPH 1998 Conference Proceedings, pages 33-42, 1998. 


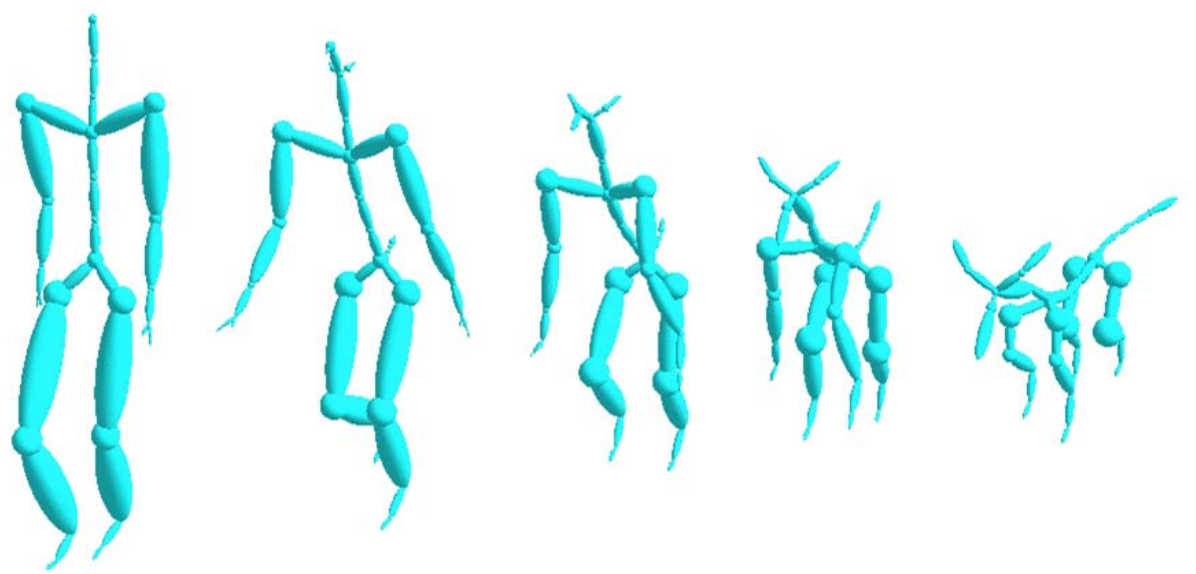

Figure 9: Concatenate a man's walking motion with a dog's walking motion.

[6] Michael Gleicher, Hyun Joon Shin, Lucas Kovar, and Andrew Jepsen. Snap-together motion: Assembling run-time animations. In Proceedings of 2003 ACM SIGGRAPH Symposium on Interactive $3 D$ graphics, pages 181-188, 2003.

[7] Lucas Kovar, Michael Gleicher, and Frèdèric Pighin. Motion graphs. ACM Transactions on Graphics (SIGGRAPH 2002 Conference Proceedings), 21(3):473482, 2002.

[8] Jehee Lee, Jinxiang Chai, Paul S. A. Reitsma, Jessica K. Hodgins, and Nancy S. Pollard. Interactive control of avatars animated with human motion data. ACM Transactions on Graphics (SIGGRAPH 2002 Conference Proceedings), 21(3):491-500, 2002.

[9] Jehee Lee and Sung Yong Shin. A hierarchical approach to interactive motion editing for human-like figures. In ACM SIGGRAPH 1999 Conference Proceedings, pages 39-48, 1999.

[10] Zoran Popovic̀ and Andrew Witkin. Physically based motion transformation. In ACM SIGGRAPH 1999 Conference Proceedings, pages 11-20, 1999.

[11] Munetoshi Unuma, Ken Anjyo, and Ryozo Takeuchi. Fourier principles for emotion-based human figure animation. In ACM SIGGRAPH 1995 Conference Proceedings, pages 91-96, 1995.

[12] Andrew Witkin and Zoran Popovic̀. Motion warping. In ACM SIGGRAPH 1995 Conference Proceedings, pages 105-108, 1995.

[13] Yonghong Zhao, Hong Yang Ong, Tiow Seng Tan, and Yongguan Xiao. Interactive control of componentbased morphing. In Proceedings of 2003 ACM SIG-
GRAPH/Eurographics Symposium on Computer Animation, pages 339-348, 2003. 